\title{
Complete cervical or thoracic spinal cord transections delay gastric emptying and gastrointestinal transit of liquid in awake rats
}

\author{
FAA Gondim ${ }^{1}$, HMP Alencar ${ }^{1}$, CL Rodrigues ${ }^{1}$, JRV da Graça $^{1}$, AA dos Santos ${ }^{1}$ and FH Rola*,1 \\ ${ }^{1}$ Departamento de Fisiologia e Farmacologia, Universidade Federal do Ceará, Fortaleza, Ceará, Brasil
}

\begin{abstract}
Study Design: To determine the changes on gastric emptying and gastrointestinal transit of liquid throughout the first week after spinal cord transection (SCT) in rats.

Methods: Male Wistar rats $(n=121)$ were fasted for $16 \mathrm{~h}$ and a complete SCT or laminectomy was performed between $C_{7}$ and $T_{1}$ (cervical group) or between $T_{4}$ and $T_{5}$ (thoracic group). Dye recovery in the stomach, proximal, mid and distal small intestine was determined $30 \mathrm{~min}, 6 \mathrm{~h}, 1,3$ or 7 days after surgery. The test meal $(1.5 \mathrm{ml}$ of a phenol red solution, $0.5 \mathrm{mg} / \mathrm{ml}$ in $5 \%$ glucose) was intragastrically administered and the animals sacrificed by cervical dislocation 10 min later.

Results: Cervical SCT increased dye recovery in the stomach $(P<0.05)$ by $70.1,78.7,34.2$, 41.3 and $50.9 \%$ while it decreased recovery in the mid small intestine $(P<0.05)$ by $87.1,85.1$, $74.8,59.5$ and $80.1 \%$, respectively $30 \mathrm{~min}, 6 \mathrm{~h}, 1,3$ and 7 days after SCT. Thoracic SCT increased gastric recovery $(P<0.05)$ by $43.5,67.6,51.2,75.4$ and $38.9 \%$ while it decreased recovery in the mid small intestine $(P<0.05)$ by $100,100,45.6,100$ and $66.6 \%$, respectively $30 \mathrm{~min}, 6 \mathrm{~h}, 1,3$ and 7 days after SCT. A separate group was submitted to laminectomy + bilateral sciatic nerve transection (paraplegic sham). Gastric emptying and gastrointestinal transit were not inhibited in this group.

Conclusion: In summary, gastric emptying and gastrointestinal transit of liquid are inhibited throughout the first week after high SCT in awake rats.
\end{abstract}

Keywords: spinal cord injury; gastrointestinal motility; gastric emptying; gastrointestinal transit; rats

\section{Introduction}

Spinal cord injury (SCI) determines severe disturbances in homeostatic mechanisms. Gastrointestinal (GI) complications, previously underestimated, are becoming more evident as a result of the improvement in postinjury care and respiratory/genitourinary management $^{1}$ and have been reported to account for $10 \%$ of fatalities in SCI patients. ${ }^{2}$

Delayed large bowel transit, ${ }^{3}$ colon and anorectal functional alterations ${ }^{4}$ have been described and large bowel dysfunction is burdensome for SCI patients. ${ }^{5}$ Reduced motility of the distal colon has also been observed in rats. ${ }^{6}$ However, there are few and yet conflicting studies (both in humans or experimental animals) addressing the disturbances on upper GI tract motility. ${ }^{7-16}$

In humans, the SCI effect on gastric emptying (GE) is still controversial, since delayed GE has been reported $^{8-10,14,16}$ but subsequently questioned. ${ }^{11,12} \mathrm{~A}$ possible explanation for such discrepancies is the fact

*Correspondence: FH Rola, Laboratório Escola Prof Luís Capelo, Departamento de Fisiologia e Farmacologia, Universidade Federal do Ceará, Rua Coronel Nunes de Melo, 1127, CEP 60.430-270, Caixa Postal 3157, Fortaleza, CE, Brazil that human studies included patients with important differences in age, sex, interval after injury, pattern (complete or partial) and injury mechanism.

In a preliminary study, we have observed that acute cervical spinal cord transection (SCT) decreases GE, intestinal and GI transit of liquid in awake rats. ${ }^{13}$ The aim of this study is thus to investigate the disturbances of GE and GI transit of liquid in awake rats, throughout the first week after complete, standard high SCT, between $\mathrm{T}_{4}$ and $\mathrm{T}_{5}$ or between $\mathrm{C}_{7}$ and $\mathrm{T}_{1}$. Part of this work has been reported in abstract form. ${ }^{17}$

\section{Materials and methods}

Surgical procedures, study design and daily care Experiments were performed on 121 male Wistar rats, weighing $160-210 \mathrm{~g}$. All surgical procedures and animal treatments were conducted in accordance with the 'Guide for the Care and Use of Laboratory Animals' DHEW Publication No (NIH) 85-23 (Bethesda, MD, USA). GE and GI transit were measured $30 \mathrm{~min}, 6 \mathrm{~h}, 1,3$ and 7 days after surgery. The animals were deprived of food $16 \mathrm{~h}$ before surgery 
but water was allowed ad libitum. They were anaesthetized with ether and after laminectomy, the seventh cervical $\left(\mathrm{C}_{7}\right)$ and first thoracic $\left(\mathrm{T}_{1}\right)$ or the fourth $\left(\mathrm{T}_{4}\right)$ and fifth thoracic $\left(\mathrm{T}_{5}\right)$ vertebrae were carefully exposed via a midline dorsal incision and the SCT performed, using a fine cut scissor. Shamoperated animals also underwent laminectomy but without subsequent cord transection. ${ }^{6}$ Bleeding was minimal and usually stopped in $10-15 \mathrm{~s}$. The completeness of the SCT was verified by careful inspection of the lesion with the aid of a $10 \times$ lens coupled to an optic light. A complete transection was confirmed in all cases and the other clinical parameters (paraplegia, lack of nociception and somatic reflexes below the lesion, as well as urinary retention) indicating completeness of the SCT were observed after anaesthesia recovery. ${ }^{18}$ Immediately after SCT, the rats were allowed to recover on a warm pad and were closely monitored for signs of respiratory or circulatory distress. Typically, the rats were awake and mobile (moving forelimbs) approximately 20 min after surgery. We considered healthy spinal rats those animals that exhibited grooming and exploratory behaviour when removed from their cage, according to Osborn et al. ${ }^{18}$

For the long term studies (1-7 days after surgery) animals received an ip antibiotic injection (procain penicillin, $30,000 \mathrm{UI} / \mathrm{kg}$ ) once a day. Animals were also placed in separate cages and maintained on rat laboratory chow and water ad libitum. Laboratory chow was broken into small pieces during the first few days to help the animals during feeding. Animals started spontaneous oral intake (food and water ingestion) on the first day after surgery. The spinalcord transected animals received regular skin and bladder care. Bladder emptying was accomplished by manual compression three to four times a day. ${ }^{19}$ Animals also received a sc saline injection $(5 \mathrm{ml}) 6 \mathrm{~h}$ after surgery to provide initial hydration. At noon time, we daily weighed the animals submitted to the 7day study.

To evaluate the effects of paraplegia per se (without the presence of spinal shock due to spinal cord transection), a separate group of sham operated animals was prepared (paraplegic sham operated animals). After performing bilateral lumbar incisions, these animals were submitted to bilateral sciatic nerve section followed by thoracic laminectomy.

\section{Measurement of GE and GI transit}

For GE and GI transit measurements, we used a modification of the technique described by Reynell and Spray. ${ }^{20}$ Animals were deprived of food $24 \mathrm{~h}$ before GE and GI transit measurements but water was allowed ad libitum until $2 \mathrm{~h}$ before the measurements.

First, $1.5 \mathrm{ml}$ of the test meal containing a nonabsorbable marker $(0.5 \mathrm{mg} / \mathrm{ml}$ phenol red solution in $5 \%$ glucose) was given orally into the stomach through a stainless steel tube that was removed immediately after delivering the solution intragastrically. The animals were sacrificed by cervical dislocation $10 \mathrm{~min}$ after test meal administration. The stomach and small intestine were exposed by laparotomy, quickly clamped at the pylorus, cardia and terminal ileum and then removed. The stomach and small intestine from the gastroduodenal junction to the caecum were carefully stretched along a meterstick on a plain top table and divided into the following segments: 1 stomach, 2 proximal $40 \%$ of small intestine, $3 \mathrm{mid} 30 \%$ of small intestine and 4 distal $30 \%$ of small intestine.

Each segment was placed in a measuring cylinder and the volume measured by adding $100 \mathrm{ml}$ of $0.1 \mathrm{~N}$ $\mathrm{NaOH}$. They were cut in small pieces and homogenized for $30 \mathrm{~s}$. The suspension was allowed to settle for $20 \mathrm{~min}$ at room temperature and $10 \mathrm{ml}$ of the supernatant was centrifuged for $10 \mathrm{~min}(2800 \mathrm{rpm})$. Proteins in $5 \mathrm{ml}$ of homogenate were precipitated with $0.5 \mathrm{ml}$ of trichloroacetic acid (20\% wt:vol), centrifuged for $20 \mathrm{~min}(2800 \mathrm{rpm})$ and $3 \mathrm{ml}$ from the supernatant was added to $4 \mathrm{ml}$ of $0.5 \mathrm{~N} \mathrm{NaOH}$. The absorbance of the sample was read at a wave length of $560 \mathrm{~nm}$ and expressed in optic densities (OD). In each experiment, a standard dilution curve was obtained relating the concentration of phenol red to the optical density of the solution in $0.1 \mathrm{~N} \mathrm{NaOH}$ solution. The linear coefficient of the standard dilution curve $(\alpha)$ was established and used to determine the concentration $(\mathrm{C})$ of the solution read at $560 \mathrm{~nm}(\mathrm{C}=\mathrm{OD})$ and subsequently the amount of phenol red $(\mathrm{m})$ recovered from each segment ( $\mathrm{m}=\mathrm{C} \times$ volume $)$.

The fractional recovery of phenol red in each segment ( $\mathrm{x}$ ) was expressed as a percentage according to the following formula:

Recovery in the segment $\mathrm{x}=$ (amount of phenol red recovered in the segment $\div$ total amount of phenol red recovered from all four segments) $\times 100$.

\section{Cardiovascular parameters}

Mean arterial pressure (MAP) and heart rate (HR) were monitored before, during and after cervical or thoracic SCT. For this purpose, in a separate group, the right carotid artery was cannulated. The carotid cannula was connected to a Narco pressure transducer (P1000B) which was coupled to a Mark IV Physiograph (Narco Byo-Systems, Houston, TX, USA).

\section{Statistical-analysis}

The results are expressed as mean \pm SEM. The one-way analysis of variance (ANOVA) and the Bonferroni's test were used to compare the differences in $\mathrm{GE}$ rates between the various groups. The ANOVA analysis of variance for repeated measures followed by the Dunnett's test were used to compare differences in MAP, HR and weight before and after cervical or 
thoracic SCT. Differences were considered significant at $P<0.05$

\section{Results}

Figures 1 and 2 show that GE and GI transit of liquid at $10 \mathrm{~min}$ after test meal administration were significantly decreased $30 \mathrm{~min}, 6 \mathrm{~h}$ and 1, 3 and 7 days after surgery in cervical or thoracic spinal cord transected animals $(P<0.05)$.

Recovery of phenol red in the stomach increased by $70.1,78.7,34.2,41.3$ and $50.9 \%$ in cervical spinal cord transected animals, respectively $30 \mathrm{~min}, 6 \mathrm{~h}, 1,3$ and 7 days after cervical SCT: from 50.5 $\pm 4.3,40.4 \pm 3.5$, $43.8 \pm 1.4,40.9 \pm 3.2$ and $42.4 \pm 4.3 \%$ in sham-operated rats $(n=5,6,5,4$ and 5) versus $85.9 \pm 6.2,72.2 \pm 2.5$, $58.8 \pm 3.3,57.8 \pm 4.3$ and $64 \pm 7.6 \%$ in cervical spinal transected animals $(n=5,6,6,5$ and $6, P<0.05)$. However, recovery in the proximal small intestine segment after cervical SCT was not significantly modified: from $30.9 \pm 2.2, \quad 40.2 \pm 7.4, \quad 31.2 \pm 1$, $37.6 \pm 2.7$ and $28.6 \pm 1.6 \%$, in sham-operated animals versus $11.7 \pm 4.9,24.9 \pm 3.6,34.9 \pm 3.9,33.5 \pm 1.3$ and $30.2 \pm 6.9 \%$ in cervical spinal transected rats $(P>0.05)$. In contrast to the stomach, we observed decreased recovery in the mid small intestine by 87.1,
$85.1,74.8,59.5$ and $80.1 \%(P<0.05)$, respectively $30 \mathrm{~min}, 6 \mathrm{~h}, 1,3$ and 7 days after SCT: $18.6 \pm 4.7$, $19.4 \pm 4.6, \quad 25 \pm 2.4, \quad 21.5 \pm 3.2$ and $29.1 \pm 4.2 \%$ in sham-operated animals versus $2.4 \pm 2.4,2.9 \pm 1.8$, $6.3 \pm 3.1,8.7 \pm 5.4$ and $5.8 \pm 2.7 \%$ in cervical spinal transected rats $(P<0.05)$.

Recovery of phenol red in the stomach increased by $43.5,67.6,51.2,75.4$ and $38.9 \%$ in thoracic spinal cord transected animals, respectively $30 \mathrm{~min}, 6 \mathrm{~h}, 1,3$ and 7 days after thoracic SCT: from $51 \pm 4.4,40.1 \pm 4.2$, $38.9 \pm 3.8,41.5 \pm 3.8$ and $41.9 \pm 2.9 \%$ in sham-operated rats $(n=5,6,5,4$ and 5$)$ versus $73.2 \pm 2.3,67.2 \pm 4.3$, $58.8 \pm 3.3,72.8 \pm 5.5$ and $58.2 \pm 7.6 \%$ in thoracic spinal transected animals $(n=5,6,6,5$ and $6, P<0.05)$. However, recovery in the proximal small intestine segment after thoracic SCT was not significantly modified: $31.1 \pm 2.1, \quad 42.7 \pm 8.6, \quad 42 \pm 8.8, \quad 32.2 \pm 3.1$ and $27.9 \pm 1.5 \%$ in sham-operated animals versus $26.8 \pm 3, \quad 32.7 \pm 8.6, \quad 30.8 \pm 6.3, \quad 27.3 \pm 5.5 \quad$ and $31.7 \pm 1.7 \%$ in thoracic spinal transected rats $(P>0.05)$. In contrast to the stomach, we observed decreased recovery in the mid small intestine by 100 , $100,45.6, \quad 100$ and $66.6 \% \quad(P<0.05)$, respectively $30 \mathrm{~min}, 6 \mathrm{~h}, 1,3$ and 7 days after SCT: $17.9 \pm 4.6$, $17.2 \pm 5, \quad 19.1 \pm 5.6, \quad 26.3 \pm 1.9$ and $30.2 \pm 2.9 \%$ in sham-operated animals versus $0 \pm 0,0 \pm 0,10.4 \pm 2.4$,

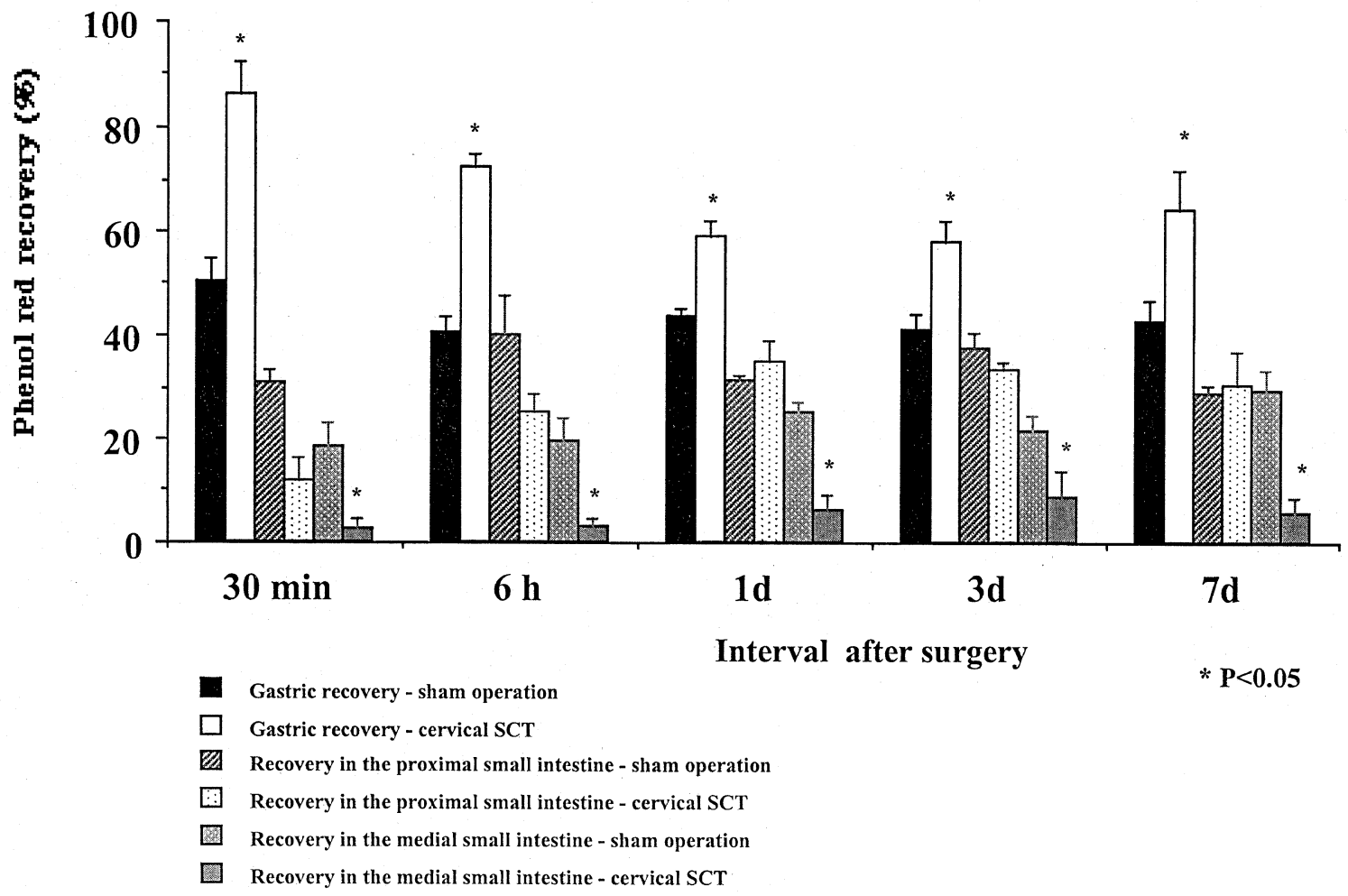

Figure 1 Comparison of the effects of cervical spinal cord transection (SCT) between $\mathrm{C}_{7}$ and $\mathrm{T}_{1}$ and sham laminectomy (sham) on the fractional $(\%)$ dye recovery from the stomach, proximal and mid small intestine segments in awake rats. The animals were studied $30 \mathrm{~min}, 6 \mathrm{~h}, 1,3$ and 7 days after surgery and sacrificed $10 \mathrm{~min}$ after test meal administration $(1.5 \mathrm{ml}$ of a $0.5 \mathrm{mg} / \mathrm{ml}$ phenol red solution in $5 \%$ glucose). ${ }^{*} P<0.05$ Bonferroni's test 


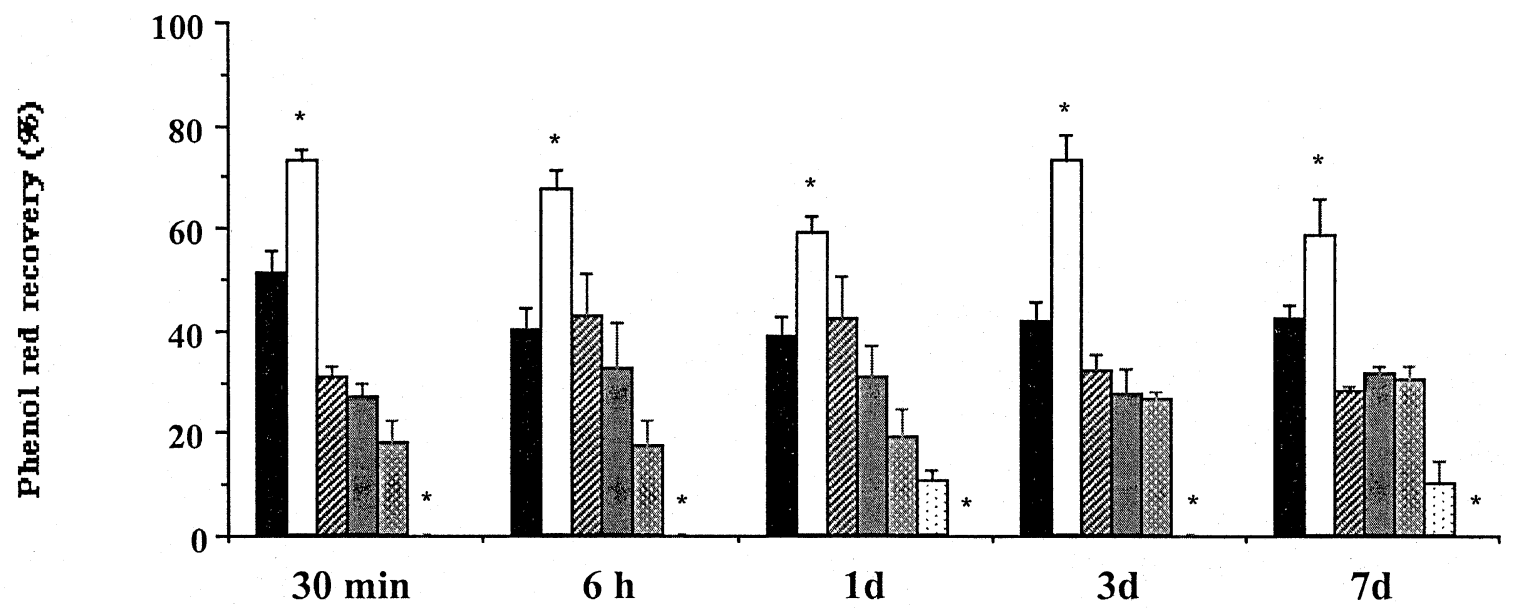

Interval after surgery

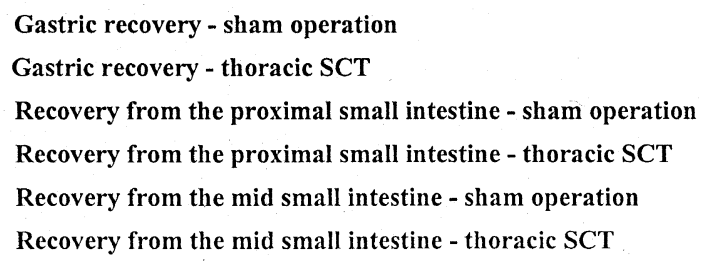

Figure 2 Comparison of the effects of thoracic spinal cord transection (SCT) between $\mathrm{T}_{4}$ and $\mathrm{T}_{5}$ and sham laminectomy (sham) on the fractional $(\%)$ dye recovery from the stomach, proximal and mid small intestine segments in awake rats. The animals were studied $30 \mathrm{~min}, 6 \mathrm{~h}, 1,3$ and 7 days after surgery and sacrificed $10 \mathrm{~min}$ after test meal administration $(1.5 \mathrm{ml}$ of a $0.5 \mathrm{mg} / \mathrm{ml}$ phenol red solution in $5 \%$ glucose). $* P<0.05$ Bonferroni's test

$0 \pm 0$ and $10.1 \pm 4.4 \%$ in thoracic spinal transected rats $(P<0.05)$.

Gastric recovery in paraplegic sham-operated animals (bilateral sciatic nerve section) was not statistically different from gastric recovery in cervical or thoracic sham-operated animals: $51.0 \pm 4.4$, $42.5 \pm 5.6, \quad 51.8 \pm 4.4, \quad 35.2 \pm 2.6$ and $30.4 \pm 2.1 \%$, respectively for $30 \mathrm{~min}, 6 \mathrm{~h}, 1,3$ and 7 days after surgery ( $n=4,4,4,4$ and 4$)$. Recovery in the proximal and mid small intestine was also not statistically significant: $31.3 \pm 5.3,37.4 \pm 2.2,31.4 \pm 5.3,42.1 \pm 3.2$ and $31 \pm 2.4$ (proximal small intestine) and $17.2 \pm 1.8$, $20.8 \pm 7.6,17.3 \pm 1.7,22.8 \pm 5.4$ and $38.6 \pm 4.3 \%$ (mid small intestine).

Figure 3 shows the daily weight variations in shamoperated animals and cervical and thoracic spinal transected animals during the 7-day observation period. As can be observed, there was a significant decrease in mean weight in spinal transected animals during the initial days after SCT. However, the weight was subsequently stable, until the animals were deprived of food for GE and GI transit measurements. Sham-operated animals gained weight from the second to the sixth day. The weight decrease in shamoperated animals from the first to the second day was because we deprived these animals of food for $12 \mathrm{~h}$ to simulate the decrease in food intake in spinal transected animals following surgery.

Cervical SCT significantly decreased MAP values from $106.3 \pm 3.9$ (pre-injury levels) to $69.6 \pm 2.8$, $64.9 \pm 2.8,68.8 \pm 2.2$ and $75.9 \pm 4.1 \mathrm{mmHg}(P<0.05)$, respectively $30 \mathrm{~min}, 6 \mathrm{~h}, 1$ and 3 days after anaesthesia recovering. MAP levels were also decreased after thoracic SCT: from $104.7 \pm 3.6 \mathrm{mmHg}$ (pre-injury levels) to $71.3 \pm 2.4, \quad 65.1 \pm 2.7, \quad 69.5 \pm 2.3$ and $76.4 \pm 3.7 \mathrm{mmHg}(P<0.05)$, respectively $30 \mathrm{~min}, 6 \mathrm{~h}$, 1 and 3 days after anaesthesia recovering. However, MAP levels returned to pre-injury levels 7 days after cervical and thoracic SCT: $95.9 \pm 4.2$ and $97.9 \pm 3.1 \mathrm{mmHg} \quad(P>0.05)$. Heart rate was also decreased after cervical and thoracic SCT from $417.9 \pm 9.5$ to $347.8 \pm 9.2, \quad 354.5 \pm 6.9, \quad 359 \pm 7.8$, $368.4 \pm 7.3$ and $376.5 \pm 5.8$ beats $/ \mathrm{min} \quad(P<0.05)$ and from $432.9 \pm 9.8$ to $353.9 \pm 7.2,349.7 \pm 6.9,358.2 \pm 9.2$, $363.5 \pm 6.3$ and $379.5 \pm 6.5$ beats $/ \mathrm{min} \quad(P<0.05)$, respectively for $30 \mathrm{~min}, 6 \mathrm{~h}, 1,3$ and 7 days after SCT.

\section{Discussion}

To the best of our knowledge, this is the first report in experimental animals describing inhibition of both GE and GI transit of liquid throughout the first week after 


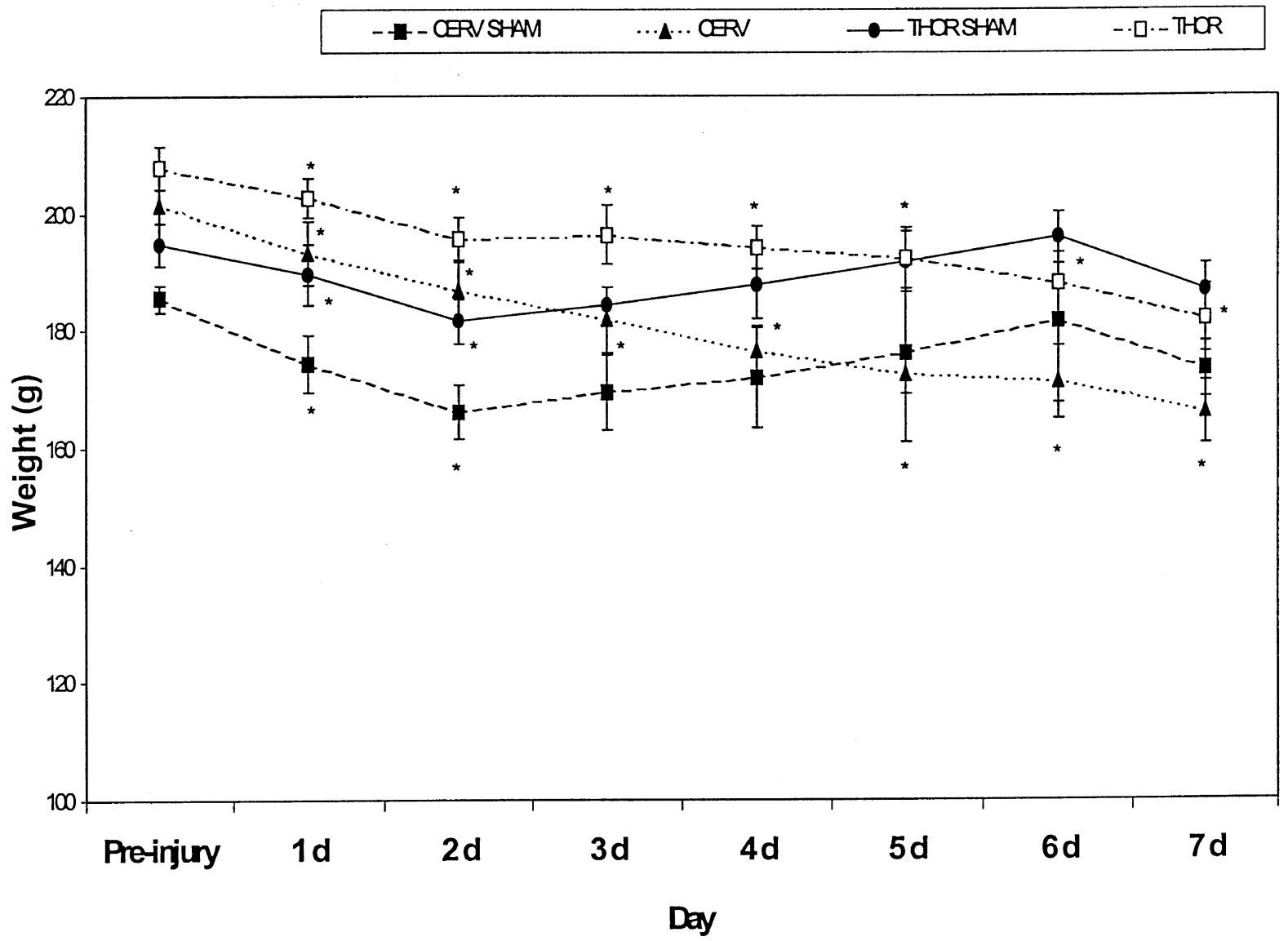

Figure 3 Daily weight changes 7 days after cervical (CERV) or thoracic (THOR) spinal cord transections as well as after thoracic or cervical sham laminectomy (THOR SHAM and CERV SHAM respectively). $* P<0.05$ Dunnett's $t$ test CERV versus CERV SHAM and THOR versus THOR SHAM

standard high cervical or thoracic spinal cord transections, between $\mathrm{C}_{7}$ and $\mathrm{T}_{1}$ or $\mathrm{T}_{4}$ and $\mathrm{T}_{5}$.

The protocol of SCT we utilized seems to be suitable to study the subacute effects of SCT on GI motility, since during the first week, the animals were stable, spontaneously ingested food and water, were active, mobile and used their forelimbs. This is in agreement with previous studies in thoracic and cervical spinal rats, which demonstrated that thoracic and cervical spinal transected animals can be studied for prolonged periods of time in healthy conditions. 18,19

In a preliminary study, we have observed that acute cervical spinal cord transection (SCT) decreases GE, intestinal and GI transit of liquid in awake rats. ${ }^{17}$ However, the animals were studied only during the first day after SCT and were also submitted to bladder catheterization. ${ }^{17}$ Afterwards, we have observed, in agreement with previous studies, ${ }^{19}$ that bladder catheterization is not essential for the daily management of spinal animals, since bladder care can be easily accomplished by expression of urine by abdominal compression three to four times a day. ${ }^{19}$
In humans, scarce and conflicting data about the effects of SCT on the motility of the upper portions of the GI tract are available: delayed GE of liquids or solids has been reported in SCI patients ${ }^{8-10,14,16}$ and subsequently questioned. ${ }^{11,12}$ A possible explanation for these discrepancies is the significant variation in age, sex, interval after injury, pattern and injury mechanism in human studies. These variations were minimized in the present study since we utilized male rats with similar age and weight and the pattern of spinal cord injury can be easily reproduced and standardized in rats.

During the interdigestive state, the stomach as well as the small intestine exhibit cyclic periods of quiescence followed by intense motor activity. ${ }^{21}$ However, the meal administration causes a rapid and marked change in the motility of the upper GI tract. Reduction in fundic tone is started and the small intestine develops patterns of irregular motor activity, resembling the phase II of the migrating myoeletric complex (MMC), which is known as fed or digestive pattern. ${ }^{21}$ The disruption of the MMC by gavage has been reported to last between 105.6 and $110.1 \mathrm{~min}$ 
after the administration of a meal containing glucose, fat and proteins. ${ }^{22}$ In rats, irregular spiking in the small intestine can be obtained after feeding with hyperosmolar saline, amino acids, oleic acid and glucose. ${ }^{23}$ The technique chosen for GE and GI transit measurements based on the recovery of phenol red, has been extensively employed in the medical literature. ${ }^{19}$ The phenol red itself appears to have a limited effect on GI motility ${ }^{23}$ and thus the technique allows the study of the motility of the GI tract during the fed state (gastric emptying and gastrointestinal transit).

The inhibition of the GE and GI transit we observed after cervical or thoracic SCT was evident not only during the first few hours $(30 \mathrm{~min}$ and $6 \mathrm{~h}$ after SCT) but also throughout the first week after SCT. During the spinal shock phase, these modifications have been ascribed to the sudden autonomic modifications elicited by SCT, which to date are not fully understood. ${ }^{24}$ Facilitation of the enterogastric ${ }^{25}$ and intestino-intestinal reflexes ${ }^{26,27}$ is a possibility since there is a sudden lack of modulation from the CNS above the level of the spinal cord injury. It is still a reasonable explanation for the inhibition after the first day post-injury since the lack of supraspinal modulation persists after the recovery from the spinal shock phase.

After the first day, the development of autonomic hyperreflexia after gavage could cause inhibition of GI transit and GE of liquid. This could be a reasonable explanation since spinal systems appear to be very active minutes after transection in rats $^{28}$ and autonomic hyperreflexia is completely established within 1 day after SCT. ${ }^{18}$ In fact, this possibility has been previously proposed, ${ }^{8}$ although it was never fully evaluated.

The decreased physical activity after spinal cord transection could also contribute to the inhibition of the GI motility we have observed throughout the first week after SCT. However, this does not appear to explain our results, since the animals from our paraplegic sham group (bilateral sciatic nerve section) did not exhibit the marked GE and GI transit changes observed after complete spinal cord transection.

We have also observed that MAP levels were significantly decreased immediately after cervical or thoracic SCT, and this fall persisted in the first 3 days after SCT, in agreement with previous results. ${ }^{18}$ However, MAP levels were not statistically different from pre-injury levels 7 days after SCT. In contrast, heart rate was significantly decreased immediately after the lesion and bradycardia persisted 7 days after SCT.

In summary, our results indicated that GE and GI transit of liquid were both inhibited during the first week after complete SCT between $\mathrm{C}_{7}$ and $\mathrm{T}_{1}$ or $\mathrm{T}_{4}$ and $\mathrm{T}_{5}$. This inhibition does not appear to be determined by decreased physical activity following SCT. Further studies are necessary to outline the mechanisms involved in this phenomenon.

\section{Note added in proof}

This work is part of a MSc thesis on Pharmacology presented by $\mathrm{F}$ de Assis Aquino Gondim to the Departamento de Fisiologia e Farmacologia, Universidade Federal do Ceará, Fortaleza, CE, Brasil. It has been presented at the 9th European Symposium on Neurogastroenterology and Motility (Maastricht, Holland, October 28-31, 1998).

\section{Acknowledgements}

We thank Dr GB Viana and Dr MR Vale for kindly providing access to laboratory facilities. This study was supported with scientific research scholarships by CAPES, CNPq, FUNCAP and UFC.

\section{References}

1 Gore RM, Mintzer RA, Calenoff L. Gastrointestinal complications of spinal cord injury. Spine 1981; 6: $538-544$.

2 Miller LS, Staas WE, Herbison GJ. Abdominal problems in patients with spinal cord lesions. Arch Phys Med Rehabil 1978; 56: $405-408$.

3 Menardo G et al. Large-bowel transit in paraplegic patients. Dis Colon Rectum 1987; 30: $924-928$.

4 Longo WE, Ballantyne GB, Modlin IM. The colon, anorectum, and spinal cord patient. A review of the functional alterations of the denervated hindgut. Dis Colon Rectum 1989; 32: 261 - 267.

5 Glickman S, Kamm MA. Bowel dysfunction in spinal-cordinjury patients. Lancet 1996; 347: 1651-1653.

6 Meshkinpour H, Harmon D, Thompson R, Yu J. Effects of thoracic spinal cord transection on colonic motor activity in rats. Paraplegia 1985; 23: $272-276$

7 Bueno L, Ferre J, Ruckebusch Y. Effect of anaesthesia and surgical procedures on intestinal myoeletric activity in rats. $\mathrm{Am} \mathrm{J}$ Dig Dis 1978; 23: 690-695.

8 Fealey RD, Szurszewski JH, Merritt JL, DiMagno EP. Effect of traumatic spinal cord transection on human upper gastrointestinal motility and gastric emptying. Gastroenterol 1984; 87: $69-$ 75.

9 Segal JL et al. Metoclopramide-induced normalization of impaired gastric emptying in spinal cord injury. $A m J$ Gastroenterol 1987; 82: 1143-1148.

10 Segal JL, Milne N, Brunemann SR. Gastric emptying is impaired in patients with spinal cord injury. Am J Gastroenterol 1995; 90: $466-470$

11 Rajendran SK et al. Gastrointestinal transit after spinal cord injury: effect of cisapride. Am J Gastroenterol 1992; 87: 16141617.

12 Zhang RL, Chayes Z, Korsten MA, Bauman WA. Gastric emptying rates to liquid and solids meals appear to be unaffected by spinal cord injury. Am J Gastroenterol 1994; 89: 1856-1858.

13 Gondim F de-AA et al. Decreased gastric emptying, intestinal and gastrointestinal transit of liquid after complete spinal cord transection. Braz J Med Biol Res 1998; 31: 1605-1610.

14 Kao C, Chang-Lai S, Chieng P, Yen T. Gastric emptying in male neurologic trauma. J Nuclear Medicine 1998; 39: $1798-1801$.

$15 \mathrm{Lu} \mathrm{C}$ et al. Gastric myoeletrical activity in patients with cervical spinal cord injury. Am J Gastroenterol 1998; 93: 2391-2396.

16 Yamada S. Effect of chronic cervical spinal cord injuries on gastric emptying. Nippon Shokakibyo Gakkai Zasshi 1987; 84: $2504-2512$.

17 Gondim F de AA et al. Cervical and thoracic complete spinal cord transections delay gastric emptying and gastrointestinal transit of liquid in awake rats. Neurogastroenterol Motility 1998; 10: 443 .

18 Osborn JW, Taylor RF, Schramm LP. Chronic cervical spinal cord injury and autonomic hyperreflexia in rats. Am J Physiol 1990; 258: R169-R174. 
19 Maiorov DN, Weaver LC, Krassioukov AV. Relationship between sympathetic activity and arterial pressure in conscious spinal rats. Am J Physiol 1997; 272: H625-H631.

20 Reynell PC, Spray GH. A technique for simultaneous measurement of absorption and transit of the gastrointestinal tract of the rat. J Physiol (London) 1956; 131: 452-462.

21 Mayer EA. The physiology of gastric storage and emptying. In: Johnson LR (ed). Physiology of the Gastrointestinal Tract. Third Edition. Raven Press, New York 1994, pp 929-976.

22 Ruckebusch M, Fioramont J. Electrical activity and propulsion in small intestine in fed and fasted rats. Gastroenterol 1975; 68: $1500-1508$

23 Rivière PJM, Liberge M, Murillo-Lopez D, Bueno L. Opposite central and peripheral control by endogenous opioids of intestinal motility in rats. Br J Pharmacol 1989; 98: 236-242.
24 Atkinson PP, Atkinson JLD. Spinal shock. Mayo Clin Proc 1996 71: $384-389$

25 Schapiro H, Woodward ER. Inhibition of gastric motility by acid in the duodenum. $J$ Appl Physiol 1995; 8: 21.

26 Johansson B, Langston J. Reflex influence of mesenteric afferents on renal, intestinal and muscle blood flow and on intestinal motility. Acta Physiol Scand 1964; 61: 400-412.

27 Johansson B, Jonsson O, Ljung B. Tonic supraspinal mechanisms influencing the intestino-intestinal inhibitory reflex. Acta Physiol Scand 1968; 72: 200-204.

28 Taylor RF, Schramm LP. Differential effects of spinal cord transection on sympathetic nerve activities in rats. Am J Physiol 1987; 253: R611 - R618. 